\title{
BMJ Open Side effects of COVID-19 vaccines: a systematic review and meta-analysis protocol of randomised trials
}

\author{
Kleyton Santos Medeiros, ${ }^{1,2}$ Ana Paula Ferreira Costa, ${ }^{1}$ \\ Ayane Cristine Alves Sarmento, ${ }^{1}$ Cijara Leonice Freitas, ${ }^{1}$ \\ Ana Katherine Gonçalves (1) ${ }^{3}$
}

To cite: Medeiros KS, Costa APF, Sarmento ACA, et al. Side effects of COVID-19 vaccines: a systematic review and meta-analysis protocol of randomised trials. BMJ Open 2022;12:e050278. doi:10.1136/ bmjopen-2021-050278

- Prepublication history for this paper is available online. To view these files, please visit the journal online (http://dx.doi. org/10.1136/bmjopen-2021050278).

$\overline{\mathrm{KSM} \text { and APFC }}$ are joint first authors.

Received 17 February 2021 Accepted 10 January 2022

Check for updates

C Author(s) (or their employer(s)) 2022. Re-use permitted under CC BY-NC. No commercial re-use. See rights and permissions. Published by BMJ.

${ }^{1}$ Health Sciences Postgraduate Program, Federal University of Rio Grande do Norte, Natal, Rio Grande do Norte, Brazil ${ }^{2}$ Instituto de Ensino, Pesquisa e Inovação, Liga Contra o Câncer, Natal, Rio Grande do Norte, Brazil

${ }^{3}$ Department of Obstetrics and Gynecology, Universidade Federal do Rio Grande do Norte, Natal, Brazil

\section{Correspondence to} Dr Ana Katherine Gonçalves; anakatherine_ufrnet@yahoo com.br

\section{ABSTRACT}

Introduction SARS-CoV-2 is responsible for a large number of global COVID-19 cases. Strategies such as social isolation, personal hygiene and frequent hand washing have been implemented; however, a protective vaccine is required to achieve sufficient herd immunity to SARS-CoV-2 infection to ultimately control the COVID-19 pandemic. To meet the urgent need for a vaccine, a reduction in the development schedule has been proposed from 10-15 years to 1-2 years. For this reason, this systematic review and meta-analysis protocol aims to compare the side effects, safety and toxicity of COVID-19 vaccines available globally, including their combinations. Methods and analysis We will select randomised controlled trial-type studies that evaluate the side effects of the COVID-19 vaccine. PubMed, Web of Science, Embase, CINAHL, PsycINFO, LILACS, SCOPUS, ClinicalTrials.gov, International Clinical Trials Registry Platform (ICTRP), medRxiv.org, biorxiv.org, preprints. org and the Cochrane Library will be searched for eligible studies until December 2021. Three reviewers will independently screen and select studies, assess methodological quality and extract data. A metaanalysis will be performed, if possible, and the Grading of Recommendations, Assessment, Development and Evaluations summary of findings will be presented. Ethics and dissemination This study will review published data, and thus it is unnecessary to obtain ethical approval. The findings of this systematic review will be published in a peer-reviewed journal.

PROSPERO registration number CRD42021231101.

\section{INTRODUCTION}

SARS-CoV-2 is responsible for a large number of global COVID-19 cases. It is a highly transmissible virus among humans that has become a significant public health issue. Symptoms include fever, dry cough, fatigue, shortness of breath, chills, muscle pain, headache, gastric disorders and weight loss, often leading to death. ${ }^{2}$

Strategies such as social isolation, personal hygiene and frequent hand washing have been implemented; however, a protective vaccine is required to achieve sufficient herd

\section{Strengths and limitations of this study}

- Four authors (KSM, APFC, ACAS, CLF) will select the articles independently using titles and abstracts.

- To the best of our knowledge, there are no existing reviews regarding the side effects of COVID-19 vaccines.

- The DerSimonian and Laird method may underestimate the true between-study variance, potentially producing overly narrow Cls for the mean effect. This fact is a limitation, so the collection of studies will be done with care and the assumptions of the analytical methods will be assessed.

immunity to SARS-CoV-2 infection to ultimately control the COVID-19 pandemic. ${ }^{3}$ To meet the urgent need for a vaccine, a reduction in the development schedule has been proposed from $10-15$ years to $1-2$ years. ${ }^{4}$

SARS-CoV-2 is an RNA virus with a high mutation rate, and that on the envelope surface has three important structural proteins that can be identified: spike protein $(\mathrm{S})$, envelope protein (E) and membrane protein (M). Most innovative vaccines have focused their efforts on inducing an immune response against the $\mathrm{S}$ protein. Attenuated virus vaccines are based on weakened microorganisms, effective in stimulating the immune system. The inactivated ones (dead microorganisms) are more stable than the attenuated ones, but they have a short duration of immunological memory that requires the association of adjuvants. mRNA vaccines are stable - and can be easily produced in large quantities. Vaccines against COVID-19 differ in composition and mechanism of action, which may be relevant for their safety and efficacy, being essential for the success and eradication of this infection. ${ }^{56}$ The viral vector (mRNA) vaccine encodes full-length $S$ protein ectodomains of SARS-CoV-2, which contains both $\mathrm{T}$ and $\mathrm{B}$ cell epitopes that can 
induce cellular and humoral immune responses against viral infection. ${ }^{7}$

Assessing the safety, efficacy and side effects of the vaccine is urgently needed, and has been heavily scrutinised by the leading medical agencies around the world, like the Centers for Disease Control and Prevention and the Food and Drug Administration. Developing any vaccine needs to ensure that safety risks are identified and quantified against potential benefits. Among the potential risks raised in the context of COVID-19, vaccine development is the security and effectiveness of immune responses elicited by a vaccine. Here, this systematic review protocol aims to assess the side effects, safety and toxicity of vaccines against COVID-19.

\section{OBJECTIVES}

This systematic review and meta-analysis protocol aims to compare the side effects, safety and toxicity of COVID-19 vaccines available globally, including their combination.

\section{Review question}

What are the rates of adverse reactions (local and systemic) to COVID-19 vaccines?

\section{METHODS AND ANALYSIS}

The meta-analysis protocol follows the Preferred Reporting Items for Systematic Review and Meta-Analysis Protocols guidelines. ${ }^{89}$ This protocol is registered with the International Prospective Register of Systematic Reviews (PROSPERO).

\section{Eligibility criteria}

The inclusion criteria involved: (1) randomised controlled trial (RCT)-type studies that evaluated the side effects of the COVID-19 vaccine; (2) experiments involving human beings; (3) studies evaluating the safety, immunogenicity and efficacy parameters of the vaccines; (4) studies that presented similar vaccination protocols; (5) studies published since January 2020 until December 2021; and (6) studies published in any language.

The exclusion criteria were as follows: (1) observational studies, and (2) case reports, meeting abstracts, review papers and commentaries.

\section{Patients, intervention, comparison, outcome strategy and types of studies}

- Patients: healthy adults aged 18 years or older who were HIV negative and previously SARS-CoV-2 infection free.

- Intervention: COVID-19 vaccine or a combination of vaccines against COVID-19.

- Comparator/control: placebo.

- Outcome: safety, tolerability and immunogenicity of the COVID-19 vaccine or the combination of vaccines against COVID-19.

- Types of studies: RCTs.

\section{Information sources}

The following databases will be searched: Medline/ PubMed, Web of Science, Embase, CINAHL, PsycINFO, Latin American and Caribbean Health Sciences Literature (LILACS), SCOPUS, ClinicalTrials.gov, International Clinical Trials Registry Platform (ICTRP), medRxiv. org, biorxiv.org, preprints.org and Cochrane Central Controlled Trials Registry. Furthermore, eligible studies may also be selected from the reference lists of retrieved articles.

\section{Patient and public involvement}

The individual patient data will not be presented. A literature search will be carried out from defined databases. No patient will be involved in the study planning and application process during neither the analysis nor the dissemination of results.

\section{Search strategy}

Our keyword search will be based on Medical Subject Headings according to the following combination: (COVID-19 OR SARS-CoV-2 OR 2019-nCoV OR coronavirus) AND (vaccines OR vaccination OR COVID-19 vaccine OR SARS-CoV-2 vaccine OR BNT162 vaccine OR mRNA-1273 vaccine OR COVID-19 aAPC vaccine OR INO-4800 vaccine OR LV-SMENP-DC COVID-19 vaccine OR Ad5-nCoV vaccine OR ChAdOx1 COVID-19 vaccine OR MNA SARS-CoV-2 S1 subunit vaccines OR PittCoVacc OR Inactivated novel coronavirus 2019-CoV vaccine Vero cells OR Inactivated Vaccines OR SARS-CoV-2 inactivated vaccines OR Viral Vaccines OR Gam-COVID-Vac vaccine OR Ad26.COV2.S vaccine OR EpiVacCorona vaccine) AND (Toxicity OR Vaccine Immunogenicity OR side effects OR adverse events) AND (randomized controlled trial OR double blind method OR clinical trial) (table 1). A list of vaccines available at $\mathrm{WHO}$ was also used.

\section{Study records}

Four researchers (KSM, APFC, ACAS, CLF) performed the selection of the studies of interest. Titles and abstracts will be read independently, and duplicate studies will be excluded. The same authors analysed the selected texts to assess the compliance with the inclusion criteria. A fifth reviewer, AKG, solves the discrepancies. The flow chart of this study is shown in figure 1.

\section{Data collection process and management}

A standardised data extraction form was developed and tested. Data from each included study will be extracted independently by two reviewers (ACAS and APFC), and any subsequent discrepancies will be resolved through discussion with a third reviewer (AKG). The data extracted will include information on authors, the year of publication, study location, type of study, main objectives, population, type of vaccine, follow-up of participants, rates of systemic events, gastrointestinal symptoms, injection siterelated adverse effects and serious vaccine-related adverse events (table 2). Furthermore, participant characteristics 
Table 1 Medline search strategy

\section{Search items}

\begin{tabular}{|c|c|}
\hline 1 & COVID-19 \\
\hline 2 & SARS-CoV-2 \\
\hline 3 & 2019-nCoV \\
\hline 4 & Coronavirus \\
\hline 5 & Or/1-4 \\
\hline 6 & vaccines \\
\hline 7 & vaccination \\
\hline 8 & COVID-19 vaccine \\
\hline 9 & SARS-CoV-2 vaccine \\
\hline 10 & BNT162 vaccine \\
\hline 11 & mRNA-1273 vaccine \\
\hline 12 & COVID-19 aAPC vaccine \\
\hline 13 & INO-4800 vaccine \\
\hline 14 & $\begin{array}{l}\text { LV-SMENP-DC COVID-19 } \\
\text { vaccine }\end{array}$ \\
\hline 15 & Ad5-nCoV vaccine \\
\hline 16 & $\begin{array}{l}\text { ChAdOx1 COVID-19 } \\
\text { vaccine }\end{array}$ \\
\hline 17 & $\begin{array}{l}\text { MNA SARS-CoV-2 S1 } \\
\text { subunit vaccines }\end{array}$ \\
\hline 18 & PittCoVacc \\
\hline 19 & $\begin{array}{l}\text { Inactivated novel } \\
\text { coronavirus } 2019-\mathrm{CoV} \\
\text { vaccine Vero cells }\end{array}$ \\
\hline 20 & Inactivated Vaccines \\
\hline 21 & $\begin{array}{l}\text { SARS-CoV-2 inactivated } \\
\text { vaccines }\end{array}$ \\
\hline 22 & Viral Vaccines \\
\hline 23 & Gam-COVID-Vac vaccine \\
\hline 24 & Ad26.COV2.S vaccine \\
\hline 25 & EpiVacCorona vaccine \\
\hline 26 & Or/6-25 \\
\hline 27 & Toxicity \\
\hline 28 & Vaccine Immunogenicity \\
\hline 29 & side effects \\
\hline 30 & adverse events \\
\hline 31 & Or/27-30 \\
\hline 32 & randomized controlled trial \\
\hline 33 & double-blind method \\
\hline 34 & clinical trial \\
\hline 35 & Or/32-34 \\
\hline 36 & 5 AND 26 AND 31 AND 35 \\
\hline
\end{tabular}

(eg, mean age, gender) and results for immunogenicity will be collected.

The study authors will be contacted in case of missing data and/or to resolve any uncertainties. In addition, any additional information will be recorded. All data entries will be checked twice. If we find a set of articles with similar characteristics based on the information in the data extraction table, we will perform a meta-analysis using a random-effects model. If there are data that are not clear in some articles, the corresponding author will be contacted for possible clarification.

\section{Risk of bias in individual studies}

Three authors (KSM, ACAS, APFC) will independently assess the risk of bias in the eligible studies using the Cochrane risk-of-bias tool. ${ }^{10}$ The Risk of Bias 2 tool $^{11}$ will be used to assess the risk of bias. Bias is assessed as a judgement (high, low or unclear) for individual elements from five domains (selection, performance, attrition, reporting and others).

Data will be entered into the Review Manager software (RevMan V.5.2.3). This software allows the user to enter protocols; complete reviews; include text, characteristics of the studies, comparison tables and study data; and perform meta-analyses. For dichotomous outcomes, we extracted or calculated the OR and $95 \%$ CI for each study. In case of heterogeneity $\left(\mathrm{I}^{2} \geq 50 \%\right)$, the random-effects model will be used to combine the studies to calculate the OR and 95\% CI using the DerSimonian-Laird algorithm ${ }^{12}$.

\section{Data synthesis and analysis}

\section{Metabias}

To grade the strength of evidence from the included data, we will use the Grading of Recommendations, Assessment, Development and Evaluation ${ }^{13}$ approach. The summary of the assessment will be incorporated into broader measurements to ensure the judgement of the risk of bias, consistency, directness and precision. The quality of the evidence will be assessed based on the risk of bias, indirectness, inconsistency, imprecision and publication bias.

\section{DISCUSSION}

The COVID-19 pandemic represents one of the most significant global public health crises of this generation. Lockdown, quarantine, contact tracing and case isolation are suggested as effective interventions to control the epidemic; however, they may present different results in different contexts because of the specific features of the COVID-19. The lack of implementation of continued interventions or effective treatments further contributes to discovering and using effective and safe vaccines. ${ }^{14} 15$

For all these reasons, scientists worldwide entered a race to find a vaccine candidate useful in fighting the new coronavirus pandemic. Nevertheless, it is essential to note that a vaccine's production is not easy and quick. Before being released to the population, a vaccine must go through three phases of clinical trials that prove its safety and effectiveness. More volunteers are recruited at 


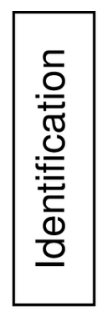

Records identified through database searches: MEDLINE ( $\mathrm{n}=\quad$ ); clinicaltrials.gov $(n=)$; Web of Science $(n=)$; EMBASE $(n=)$; CINAHL $(n=)$; LILACS $(n=)$; Scopus $(n=)$ ); Cochrane $(n=)$.
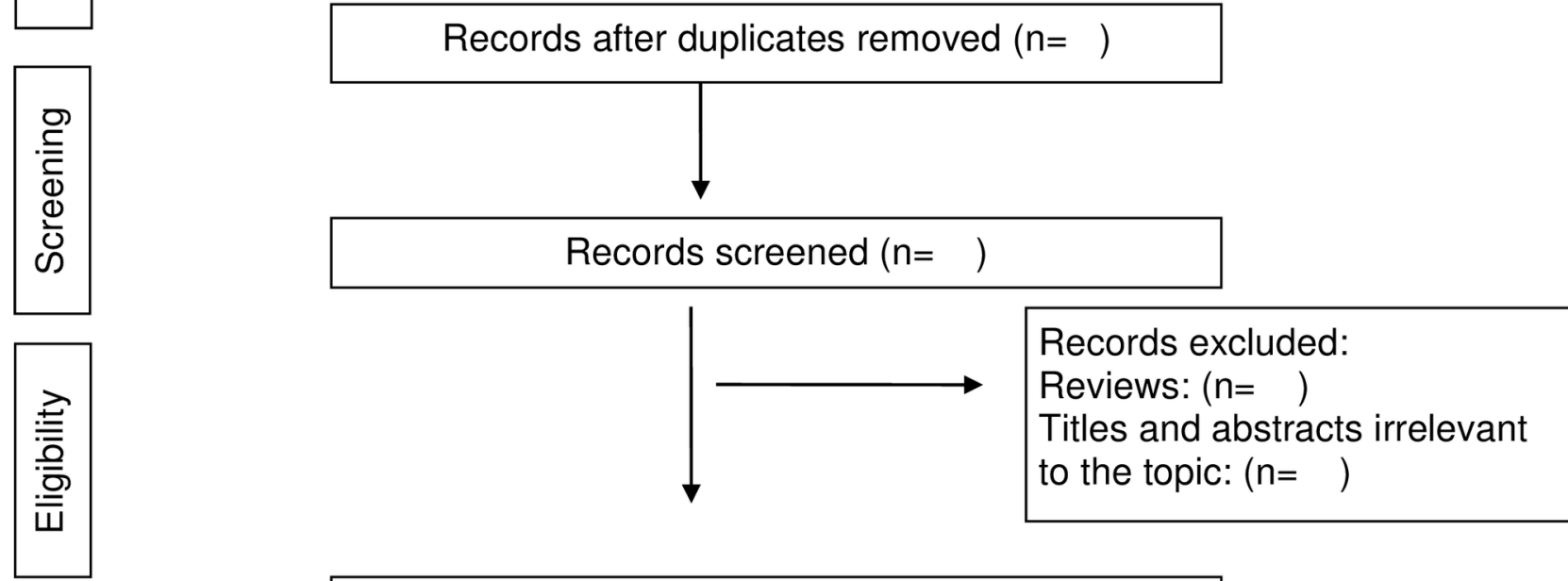

Full-text articles assessed for eligibility $(n=\quad)$
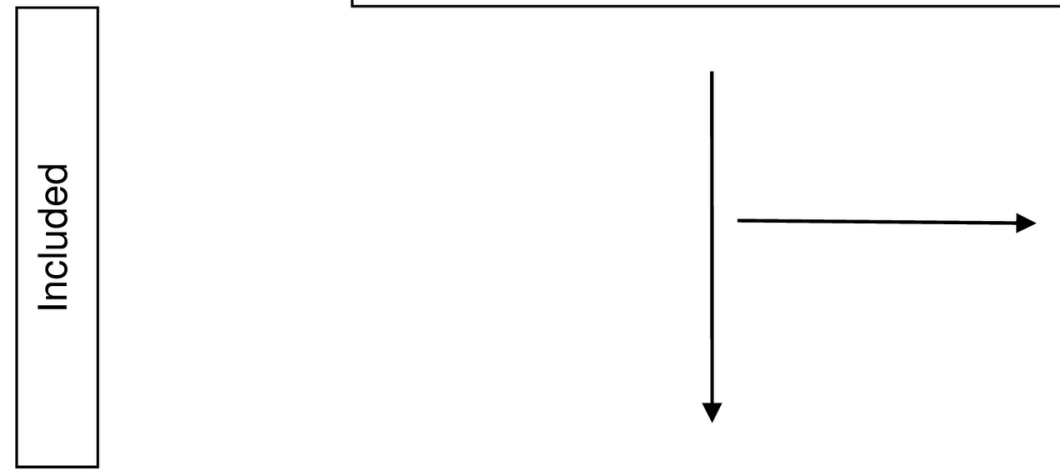

Records excluded:

Case reports: $(\mathrm{n}=$ )

Publications that are not specifically about use of the COVID19 vaccine: $(n=)$ Insufficient data to be extracted or calculated: $(n=)$

\section{Studies included in the qualitative synthesis}

$(n=)$

Studies included in the quantitative synthesis (metaanalysis) $(n=)$

Figure 1 Flow diagram of the search for eligible studies on the side effects, safety, and toxicity of the COVID-19 vaccine: CENTRAL, Cochrane Central Register of Controlled Trials.

Figure 1 Flow diagram of the search for eligible studies on the side effects, safety and toxicity of the COVID-19 vaccine. CENTRAL, Cochrane Central Register of Controlled Trials. 
Table 2 Adverse events of COVID-19 vaccines

\begin{tabular}{|c|c|}
\hline \multicolumn{2}{|l|}{ Adverse events } \\
\hline Systemic event reactions $(10,11)$ & $\begin{array}{l}\text { Fever or hyperthermia or feverish, headaches, fatigue, vomiting, diarrhoea, muscle } \\
\text { pain, joint pain, cough, nausea, dyspnoea, appetite impaired, dizziness, mucosal } \\
\text { abnormality, pruritus, }{ }^{27} 28 \text { hypersensitivity, syncope, }{ }^{27} \text { asthenia, rhinorrhoea, malaise, } \\
\text { sore throat (throat irritation), pain in the oropharynx (pharyngalgia), hives, nasal } \\
\text { congestion }{ }^{29} 30\end{array}$ \\
\hline Injection site adverse reactions (10-12) & Pain, induration, redness or erythema, swelling, itch, muscular weakness ${ }^{27-29}$ \\
\hline Serious vaccine-related adverse event & Deaths, hospitalisation, ${ }^{30}$ thrombotic complications ${ }^{31} 32$ \\
\hline
\end{tabular}

each stage, and the researchers analyse the test results to ensure that a vaccine can be licensed. ${ }^{16-18}$

One hundred and seventy-three vaccines were in preclinical development and 64 in clinical trials until 20 January 2021. On 31 December 2020, the WHO listed the mRNA vaccine against COVID-19 for emergency use, making this Pfizer/BioNTech immuniser the first to receive WHO emergency validation from the beginning outbreak. Already, in January 2021, emergency approval was granted to nine vaccines by regulatory authorities in different parts of the world. ${ }^{1419}$

With the starting vaccination, several studies were carried out to ascertain the safety of these vaccines, since they were produced in record time. ${ }^{20-22}$ Currently, one systematic review about the thematic showed that of 11 published clinical trials of COVID-19 vaccines included in the study, adverse reactions reported were considered mild to moderate with few severe reactions which were unrelated to the test vaccine. Common adverse events were pain at the site of injection, fever, myalgia, fatigue and headache. Serious adverse events (SAE) were reported in four trials: COVID-19 Vaccine AstraZeneca (AZD1222) - 168 SAEs with only three related to the vaccine; Ad26.COV2.S-four with none related to the testing vaccine; five with Comirnaty (BNT162b1) vaccine and one with Covaxin (BBV152) vaccine. ${ }^{19}$

One limitation about the COVID-19 vaccine safety tested until now is that clinical trials of the safety and effectiveness have had low inclusion of vulnerable groups, for example, older persons, the first population to receive the whole vaccine. That's why pharmacovigilance postmarketing is necessary to surveillance of new drugs, as a critical aspect of evaluating medicine safety and effectiveness, particularly in risk groups.

Other prevention approaches are likely to emerge in the coming months, including antiviral agents, drugs may be to decrease disease progression, monoclonal antibodies, hyperimmune globulin and convalescent titre. If proven effective, these approaches could be used in high-risk individuals, including healthcare workers, other essential workers and older adults. ${ }^{23-26}$ It is essential to maintain protective measures such as washing hands frequently with soap and water or gel alcohol and covering the mouth with a forearm when coughing or sneezing.
For all the reasons mentioned above, this review is necessary and essential. The latter is a well-defined protocol registered with PROSPERO, well planned to include the largest possible number of vaccines, a significant number of vaccinated patients, thus providing safe and reliable results regarding the use of vaccines.

\section{Ethics and dissemination}

This study will review published data, and thus it is unnecessary to obtain ethical approval. The findings of this systematic review will be published in a peer-reviewed journal.

Contributors KSM, ACAS and APFC contributed to the design of this review. KSM and ACAS drafted the protocol manuscript. APFC and AKG revised the manuscript. KSM, AKG and APFC developed the search strategies. KSM, CLF and ACAS implemented the search strategies. KSM, CLF, ACAS and APFC tracked the potential studies, extracted the data and assessed the quality. In case of disagreement between the data extractors, AKG advised on the methodology and worked as a referee. KSM completed the data synthesis. All authors approved the final version for publication.

Funding The authors have not declared a specific grant for this research from any funding agency in the public, commercial or not-for-profit sectors.

Competing interests None declared.

Patient and public involvement Patients and/or the public were not involved in the design, or conduct, or reporting, or dissemination plans of this research.

Patient consent for publication Not applicable.

Provenance and peer review Not commissioned; externally peer reviewed.

Open access This is an open access article distributed in accordance with the Creative Commons Attribution Non Commercial (CC BY-NC 4.0) license, which permits others to distribute, remix, adapt, build upon this work non-commercially, and license their derivative works on different terms, provided the original work is properly cited, appropriate credit is given, any changes made indicated, and the use is non-commercial. See: http://creativecommons.org/licenses/by-nc/4.0/.

ORCID iD

Ana Katherine Gonçalves http://orcid.org/0000-0002-8351-5119

\section{REFERENCES}

1 Johansson MA, Quandelacy TM, Kada S, et al. SARS-CoV-2 transmission from people without COVID-19 symptoms. JAMA Netw Open 2021;4:e2035057.

2 Kaur SP, Gupta V. COVID-19 vaccine: a comprehensive status report. Virus Res 2020;288:198114.

3 Graham BS. Rapid COVID-19 vaccine development. Science 2020;368:945-6.

4 Jeyanathan M, Afkhami S, Smaill F, et al. Immunological considerations for COVID-19 vaccine strategies. Nat Rev Immunol 2020;20:615-32. 
5 Forni G, Mantovani A. COVID-19 vaccines: where we stand and challenges ahead. Cell Death Differ 2021;28:626-39.

6 Pollard AJ. Notice of addendum to article reporting Oxford trial of ChAdOx1 nCoV-19 vaccine. The Lancet 2020;396:e89.

7 Cao M, Su X, Jiang S. Broad-Spectrum Anti-coronavirus vaccines and therapeutics to combat the current COVID-19 pandemic and future coronavirus disease outbreaks. Stem Cell Reports 2021;16:398-411.

8 Moher D, Liberati A, Tetzlaff J, et al. Preferred reporting items for systematic reviews and meta-analyses: the PRISMA statement. PLoS Med 2009;6:e1000097.

9 Liberati A, Altman DG, Tetzlaff J, et al. The PRISMA statement for reporting systematic reviews and meta-analyses of studies that evaluate healthcare interventions: explanation and elaboration. BMJ 2009;339:b2700.

10 Balshem $\mathrm{H}$, Helfand $\mathrm{M}$, Schünemann $\mathrm{HJ}$, et al. Grade guidelines: 3 . rating the quality of evidence. J Clin Epidemiol 2011;64:401-6.

11 lezadi S, Azami-Aghdash S, Ghiasi A, et al. Effectiveness of the non-pharmaceutical public health interventions against COVID-19; a protocol of a systematic review and realist review. PLoS One 2020;15:e0239554.

12 DerSimonian R, Laird N. Meta-Analysis in clinical trials. Control Clin Trials 1986;7:177-88.

13 Wiersinga WJ, Rhodes A, Cheng AC, et al. Pathophysiology, transmission, diagnosis, and treatment of coronavirus disease 2019 (COVID-19). JAMA 2020;324:782.

14 Who. coronavirus disease (COVID-19) situation report-181. Geneva: World Health organization 2020

15 Ahn D-G, Shin H-J, Kim M-H, et al. Current status of epidemiology, diagnosis, therapeutics, and vaccines for novel coronavirus disease 2019 (COVID-19). J Microbiol Biotechnol 2020;30:313-24.

16 Dhama K, Sharun K, Tiwari R. COVID-19, an emerging coronavirus infection: advances and prospects in designing and developing vaccines, immunotherapeutics, and therapeutics. Human Vaccines \&Immunotherapeutics 2020:1-7.

17 Folegatti PM, Ewer KJ, Aley PK, et al. Safety and immunogenicity of the ChAdOx1 nCoV-19 vaccine against SARS-CoV-2: a preliminary report of a phase $1 / 2$, single-blind, randomised controlled trial. Lancet 2020;396:467-78.

18 Ong E, Wong MU, Huffman A, et al. COVID-19 coronavirus vaccine design using reverse vaccinology and machine learning. Front Immunol 2020;11:1581.

19 Kaur RJ, Dutta S, Bhardwaj P. Adverse events reported from COVID-19 vaccine trials: a systematic review. Ind J ClinBiochem 2021.
20 Chen J, Cai Y, Chen Y, et al. Nervous and muscular adverse events after COVID-19 vaccination: a systematic review and meta-analysis of clinical trials. Vaccines 2021;9:939.

21 Lopez Bernal J, Andrews N, Gower C, et al. Effectiveness of the Pfizer-BioNTech and Oxford-AstraZeneca vaccines on covid-19 related symptoms, hospital admissions, and mortality in older adults in England: test negative case-control study. BMJ 2021;373:n1088.

22 Iheanacho CO, Eze UIH, Adida EA. A systematic review of effectiveness of BNT162b2 mRNA and ChAdOx1 adenoviral vector COVID-19 vaccines in the general population. Bull Natl Res Cent 2021;45:150.

23 Sanders JM, Monogue ML, Jodlowski TZ, et al. Pharmacologic treatments for coronavirus disease 2019 (COVID-19). JAMA 2020;382

24 Yuan P, Ai P, Liu Y, et al. Safety, tolerability, and immunogenicity of COVID-19 vaccines: a systematic review and meta-analysis. medRxiv 2020. doi:10.2139/ssrn.3746259. [Epub ahead of print: 04 Nov 2020].

25 Dhakal N, Poudyal A, Gyanwali P. Pharmacological treatment for the management of COVID 19: a narrative review. J Nepal Med Assoc 2021;59:614-21.

26 Bestetti RB, Furlan-Daniel R, Silva VMR. Pharmacological treatment of patients with mild to moderate COVID-19: a comprehensive review. Int J Environ Res Public Health 2021;18:7212.

27 Zhu F-C, Li Y-H, Guan X-H, et al. Safety, tolerability, and immunogenicity of a recombinant adenovirus type- 5 vectored COVID-19 vaccine: a dose-escalation, open-label, non-randomised, first-in-human trial. Lancet 2020;395:1845-54.

28 Zhu F-C, Guan X-H, Li Y-H, et al. Immunogenicity and safety of a recombinant adenovirus type-5-vectored COVID-19 vaccine in healthy adults aged 18 years or older: a randomised, double-blind, placebo-controlled, phase 2 trial. The Lancet 2020;396:479-88.

29 Logunov DY, Dolzhikova IV, Zubkova OV, et al. Safety and immunogenicity of an Rad26 and RAD5 vector-based heterologous prime-boost COVID-19 vaccine in two formulations: two open, nonrandomised phase 1/2 studies from Russia. Lancet 2020;396:887-97.

30 Folegatti PM, Ewer KJ, Aley PK, et al. Safety and immunogenicity of the ChAdOx1 $\mathrm{nCoV}-19$ vaccine against SARS-CoV-2: a preliminary report of a phase $1 / 2$, single-blind, randomised controlled trial. The Lancet 2020;396:467-78.

31 Porres-Aguilar M, Guerrero-de León MC, Grimaldo-Gómez FA. Thrombotic complications in severe COVID-19: focus on venous thromboembolism, thromboprophylaxis and anticoagulation. CirCir 2021;89:115-9.

32 Porres-Aguilar M, Lazo-Langner A, Panduro A, et al. COVID-19 vaccine-induced immune thrombotic thrombocytopenia: an emerging cause of splanchnic vein thrombosis. Ann Hepatol 2021;23:100356. 